\title{
Horizonte académico de los graduados, modalidad presencial de la Corporación Universitaria del Caribe "CECAR"
}

\section{Academic horizon of the graduates, face-to-face modality of the Caribbean University Corporation "CECAR"}

\author{
Kelly Mercedes Díaz-Theran \\ Corporación Universitaria del Caribe CECAR, Sincelejo, Colombia \\ $\triangle$ kelly.diaz@cecar.edu.co \\ D https://orcid.org/0000-0001-5893-1439 \\ Rosana Martínez-Carazo \\ Corporación Universitaria del Caribe CECAR, Sincelejo, Colombia \\ $\triangle$ rosana.martinezc@cecar.edu.co \\ (i) https://orcid.org/0000-0003-0087-8377
}

\section{Martha Lucia-Contreras}

Corporación Universitaria del Caribe CECAR, Sincelejo, Colombia

$\triangle$ martha.contrerasc@cecar.edu.co

https://orcid.org/0000-0002-6874-8459

Recibido: 12-02-2019

Aceptado: 20-06-2019

Publicado: 22-06-2019

\section{RESUMEN}

Objetivo. El factor graduado es un eje que cobra gran importancia en los procesos de formación y lineamientos de calidad de las instituciones de educación superior (IES), en pro de obtener acreditación de calidad, es por esto que los titulados se constituyen en aliados estratégicos para la interacción constante con el sector productivo y las problemáticas del entorno, haciéndose explícito que la pretensión de esta investigación es evaluar el horizonte académico de los graduados de la Corporación Universitaria del Caribe. Metodología. Investigación con enfoque cuantitativo descriptivo, de corte transversal; para la recolección de la información se utilizarán las encuestas de seguimiento a graduados y datos del Observatorio Laboral de Educación (OLE), la población está conformada por los graduados de la modalidad presencial de la Corporación Universitaria del Caribe CECAR desde el 2013 hasta el 2017 con un total de 2524 y un muestreo probabilístico estratificado de 548 graduados de los programas de modalidad presencial. Resultados. Se destaca que el $41.1 \%$ de los egresados ha realizado algún posgrado de los cuales el $27.7 \%$ corresponden a especializaciones, un $12.2 \%$ a maestrías, y el $1.1 \%$ a doctorado, además el $13.2 \%$ de los graduados de CECAR participan en comunidades académicas reconocidas del sector productivo tanto en el ámbito nacional como internacional. Conclusiones. Esta información contribuye a la ruta para efectuar ajustes curriculares a los programas académicos atendiendo a las necesidades del entorno y demandas del mercado laboral que aporten al desarrollo sostenible, convivencia y paz, producto de la formación de profesionales integrales, con capacidad de trasformar la realidad social y económica.

Palabras claves: seguimiento de graduados; empleabilidad; sector productivo. 


\section{ABSTRACT}

Objective. The graduate factor is an axis that takes great importance in the training processes and quality guidelines of higher education institutions (IES), in order to obtain quality accreditation, which is why the graduates are strategic allies for constant interaction with the productive sector and the problems of the environment, making explicit that the intention of this research is to evaluate the academic horizon of the graduates of the Caribbean University Corporation. Methodology. Research with a descriptive quantitative approach, cross-sectional, for the collection of information will use follow-up surveys of graduates and data from the Labor Observatory of Education (OLE), the population is made up of graduates of the face-to-face modality of the Caribbean University Corporation CECAR from 2013 to 2017 with a total of 2524 and a probabilistic stratified sample of 548 graduates of the face-to-face modality programs. Results. It should be noted that $41.1 \%$ of CECAR graduates have completed a postgraduate degree, of which $27.7 \%$ correspond to specializations, $12.2 \%$ to master's degrees, and $1.1 \%$ to doctoral degrees. In addition, $13.2 \%$ of CECAR graduates participate in nationally and internationally recognized academic communities in the productive sector. Conclusions. This information contributes to the route for making curricular adjustments to academic programs in response to the needs of the environment and demands of the labor market that contribute to sustainable development, coexistence and peace, as a result of the training of integral professionals with the capacity to transform social and economic reality.

Keywords: graduate follow-up; employability; productive sector.

\section{Introducción}

Los procesos institucionales de la Corporación Universitaria del Caribe - CECAR contemplan dentro de sus ejes misionales y su direccionamiento estratégico, el mejoramiento continuo que se visiona en la autoevaluación y autorregulación; en este sentido los graduados son la ventana al sector externo que permiten observar a los programas de pregrado y coadyuvar con el redireccionamiento de los distintos procesos académico administrativos y de esta forma cambiar las perspectivas del impacto que se tiene en el sector externo de un programa académico profesional, acorde a las necesidades del contexto regional y que tribute al plan de desarrollo interinstitucional.

Esto se logra, teniendo en cuenta las características de la población y el contexto donde se desarrolla la acción, según Pérez, González y Polo (2018) el estudiante de CECAR se encuentra en un nivel sociodemográfico entre 1 y 2 con ingresos salariales mensuales que no superan los 2 salarios mínimos legales, esto denota la importancia de la misión y la función social en el marco institucional de compromiso con los grupos vulnerables del departamento y la región caribe. De esta forma según Sanabria, Silveira y Diaz (2017) parece apropiado definir universidad como un sistema social, organizado para la consecución de un tipo particular de meta desde la responsabilidad social.

El estudio cuyo objetivo primordial es evaluar el reconocimiento académico y laboral de los graduados de la Corporación Universitaria del Caribe, permite establecer la participación de los titulados en comunidades académicas, asociaciones o participación de estos en el sector productivo del departamento.

Sobre la base de las anteriores consideraciones, se pretende evaluar el reconocimiento que han tenido los profesionales asociado al desempeño competitivo durante su vida laboral y conocer de primera mano la apreciación sobre la calidad de la formación 
brindada por CECAR a los graduados y si están o no acorde a las exigencias del cargo que ocupan. Es importante destacar que la información recopilada y que da origen a esta investigación, en efecto se obtienen de la fuente primaria de las encuestas y focus group a los graduados.

Desde el surgimiento de la Educación terciaria se habla de la transición de los profesionales hacia su entorno social, y para el contexto de las universidades el conocimiento con que salen los graduados, producto de la formación curricular impartida por estas, queda disponible para la sociedad. Como eje fundamental y como factor importante del proceso de Acreditación de una Institución de Educación Superior (IES), los graduados, están tomando auge dentro de la comunidad académica; pues cada vez toma más significancia que una IES, realicen seguimiento a sus graduados, con el fin de mejorar la educación que imparte a los estudiantes, atendiendo a las necesidades del entorno.

Resulta oportuno precisar algunos antecedentes a nivel internacional que han aportado a los estudios de Seguimiento a graduados, uno de ellos, el proyecto Career after Higher Education: a European Research Study (Cheers) donde un equipo de investigadores de varios países deciden realizar un estudio sobre la situación laboral de los jóvenes graduados en enseñanza superior Cheers, que hizo seguimiento a 36.000 mil egresados en tres periodos posteriores al grado, con el fin de medir sus competencias laborales. Partiendo de la premisa de que los países escogidos tuvieran un poder adquisitivo parecido; Ios análisis se efectuaron en Alemania, Austria, España, Finlandia, Francia, Holanda, Italia, Noruega, Reino Unido, República Checa, Suecia y Japón, única nación no europea evaluada (Universidad Nacional de Colombia , 2011).

Otro estudio de gran magnitud, para América Latina, es el proyecto Tuning considerado el segundo a nivel intercontinental cuyo propósito "es contribuir al desarrollo de las titulaciones fácilmente comparables y comprensibles [desde adentro], en base a los objetivos que la titulación se marque, desde los perfiles buscados para los egresados" (Beneitone, et al., 2007, p. 15). Los resultados del estudio en términos de los graduados permitieron analizar dentro del contexto, las competencias y destrezas que pueden relacionarse y ayudar a los graduados a resolver problemas en el campo de acción.

Asimismo, en Ecuador, Tirado, Tejeda, Cedeño (2015) presentan de una Universidad laica, llamada Eloi Alfaro de Manabí, el proceso de institucionalización de seguimiento a graduados dando cuenta que, es un sistema coordinado donde apoyan distintas unidades académicas la labor de ubicación y actualización de los graduados para analizar los datos y realizar los informes correspondientes. El modelo cuenta con los siguientes componentes: Objetivos, muestreo, variables de estudio, método de indagación, organización de los resultados, organización del funcionamiento, observatorio laboral para la educación superior (OLE).

En un programa de maestría en ciencias de la salud pública, de la Universidad del Oeste - Inglaterra, Bristol, Reino Unido, desarrollan un estudio sobre empleabilidad y experiencias profesionales de los graduados internacionales, un estudio secuencial de métodos mixtos entre enero y abril de 2017, calcularon estadísticas de tipo descriptivas sobre los datos obtenidos mediante encuestas y los datos cualitativos los analizan de manera temática; como resultados relevantes obtuvieron que el $63 \%$ fueron contratados después de un año de grado, 15\% continuaron estudios de doctorado, los graduados en el estudio manifestaron que están en roles o actividades que mejoraron después de su grado en áreas de análisis de políticas en salud pública, roles de liderazgo e investigación y las competencias más relevantes para los cargos actuales son: análisis crítico, pensamiento multidisciplinario, habilidades de liderazgo en salud pública. El estudio finalmente concluye la importancia de la educación superior en salud pública 
para el desarrollo de su profesión y los graduados manifestaron su satisfacción con la relevancia e impacto de las competencias que aprendieron (Buunaaisie, et al., 2018).

En el contexto nacional, se encuentra una investigación realizada en una Universidad pública del Sur Occidente Colombiano en los periodos comprendidos entre el 2009 2013 para identificar el impacto de los graduados de una especialización en fisioterapia cardiopulmonar a nivel laboral. Es un estudio descriptivo y para la aplicación de la encuesta utilizaron el correo electrónico como medio para la recolección de la información. La tasa de graduados donde se relacionaba la formación con las actividades del trabajo fue del 98\%; la actividad laboral predominante es la atención a pacientes, y el área de mejor desempeño ha sido el servicio de cuidados intensivos para adultos, el impacto positivo ha sido la mejora en atención a pacientes y el menor impacto lo tiene el salario. Los autores concluyen que la formación como profesionales de esa área, les mejora el rendimiento a los graduados y les ayuda a que apliquen dichas competencias adquiridas en su lugar de trabajo (Wilches, Muñoz, Carvajal y Segura 2016).

La universidad de Sucre - Sincelejo Colombia, desarrolló una investigación sobre el impacto de los graduados del programa de enfermería de la universidad en mención, en sus ambientes de trabajo desde la mirada de empleadores, usuarios y graduados. Un estudio de corte cualitativo, descriptivo, con un muestreo no probabilístico por conveniencias; donde participaron 25 empleadores. Los principales resultados arrojados por este estudio es la evidencia de competencias comunicativas en los egresados, y que cuentan con las habilidades de aplicar los conocimientos teóricos (Jaramillo, Montesino, Suarez, Vergara y Martinez, 2018).

A nivel local, en el año 2011, en el programa de Psicología realizó una investigación denominada "Competencias laborales requeridas a los psicólogos egresados de la Corporación Universitaria del Caribe - CECAR" participaron 50 egresados, quienes a través de un cuestionario de (Ruiz, Jaraba y Romero, 2005) dieron cuenta de los conocimientos, habilidades y actitudes que se le demandan a los profesionales de la Psicología en el mercado laboral en Sincelejo. (Paternina y Reyes, 2011). Dentro de los resultados más sobresalientes de está, se destacan las experiencias de los estudiantes en su formación, pues un programa exitoso debe brindar una infraestructura adecuada, gestión de contenidos, y recursos, oportunidades de crecimiento académico y personal, así como una interacción estudiantes- docentes de calidad. (Paternina, Chagüi, Castro y Buitrago, 2015).

Si bien hay investigaciones que denotan que se ha estudiado sobre el tema, es necesario para la Corporación Universitaria del Caribe - CECAR tener información actualizada de un estudio de impacto sobre los graduados, que permita un proceso de retroalimentación y de mejora continua que conlleven a ajustes curriculares institucionales, y cumplir con requerimientos exigidos a nivel de normatividad.

Según el acuerdo de Junta Directiva № 17 de agosto de 2014 de la Corporación Universitaria del Caribe - CECAR, se faculta a la Universidad para constituir una Unidad de Seguimiento a graduados y se conceptualiza este término y "se entiende por graduado quien haya cumplido con todos los requisitos académicos y administrativos de la Corporación para optar a un título académico y ésta le certifique su idoneidad con la expedición del diploma y del acta de grado" (Art. 1, Parágrafo Único).

Graduado es el estudiante que ha recibido el grado por parte de una Institución de Educación Superior como muestra de la culminación de su ciclo académico. Un estudiante que termina materias, pero, no ha obtenido el título es un egresado no graduado y puede ser catalogado como desertor de acuerdo con el criterio" (Ministerio de Educación Nacional, en adelante MEN). 
Para el MEN (2007), realizar seguimiento a los graduados es una tendencia creciente en países que buscan mejorar la calidad y la pertinencia de los programas académicos. Los sistemas de información suministran insumos que están usando las instituciones, el sector productivo, el gobierno y los estudiantes para tomar decisiones" (p. 1). El ámbito internacional y nacional da muestra de la necesidad de estudiar el impacto de un graduado en el medio social; así como el impacto del quehacer del graduado en el sector empresarial se volvió tema de interés y surgieron análisis que lo demuestran. Uno de los primeros lo realizó el geógrafo inglés Ron J. Johnston 1991, quien revisó el desempeño de los profesionales en el sector productivo del reino unido a partir de 1980. Este tipo de evaluaciones ha tenido en cuenta indicadores de éxito tales como: volumen de ingresos, nivel ocupacional alcanzado o la formación como persona que los propios graduados perciben haber conseguido (p. 4).

Por consiguiente, y como resultado de esta investigación se pretende aportar a la mejora continua de la calidad de la educación superior, a través del seguimiento a los graduados, estrategia que permite retroalimentar el currículo como proceso que contribuye a la formación idónea del estudiante para generar una identidad de los profesionales que egresan de la IES.

Los estudios de seguimiento a graduados permiten analizar la relación entre las competencias adquiridas con la educación superior y las requeridas por los empleadores con el fin de comprender el proceso de transición al mercado laboral de los graduados de la educación superior (OLE, 2013).

Para el estudio de seguimiento, los principales actores son los graduados. Las encuestas en sus diferentes momentos son una herramienta sugerida por el Ministerio de Educación Nacional a través del sistema de información habilitado en la página del Observatorio Laboral de la Educación, o sistemas propios de la IES que permiten adoptar un modelo de seguimiento para analizar la transición de los graduados al mercado laboral y la percepción que estos tienen sobre la Institución que los formó.

El Decreto 1075 de mayo de 2015, por el cual se expide el decreto único del sector educación, en su artículo 2.5.3.2.2.2. Sobre la evaluación de las condiciones de calidad de carácter institucional, señala la importancia de que las IES tengan un programa de seguimiento a graduados. Es por ello que se plantea desde esta normativa los lineamientos que lo definen para cualquier Institución de Educación Superior, es preciso entonces referenciar que un Programa de egresados, es el desarrollo de una estrategia de seguimiento a corto y largo plazo, que permita conocer y valorar su desempeño y el impacto social del programa, así como estimular el intercambio de experiencias académicas e investigativas (El Decreto 1075 de mayo de 2015).

Así mismo, los lineamientos para acreditación de programas, direccionan la ruta a través de la Guía de lineamientos del Sistema Nacional de Acreditación (CNA, 2013) que establece el Factor: "impacto de los graduados en el medio" expresado desde la postura que un programa de alta calidad se reconoce a través del desempeño laboral de sus graduados y del impacto que estos tienen en el proyecto académico y en los procesos de desarrollo social, cultural y económico de sus respectivos entornos. (p. 46); es por ello que el lineamiento establece dos características: una referente al seguimiento a los graduados y otra, al impacto de los graduados en el medio social y académico. 


\section{MÉTODO}

Diseño y tipo de estudio. La investigación está enmarcada bajo un paradigma positivista, puesto que se pretende conocer la realidad que puede ser observable y medible con el propósito de que los resultados estén dentro de la objetividad (Briones, 1996). El enfoque de la investigación es cuantitativo, ya que se utiliza un instrumento de medición que permiten recolectar, procesar y analizar datos de forma estructural y sistemática, en concordancia con el propósito de elaborar un conocimiento objetivo y verificable.

El tipo de estudio es descriptivo, pues detalla características, propiedades y/o rasgos del fenómeno analizado, en este caso se pretende evaluar las particularidades (Hernandez Sampieri y Mendoza Torres , 2018) y lo que hace distintivo a los graduados de CECAR; es de corte transversal, debido que se pretende estudiar el fenómeno tal como aparece en el presente en cuanto a sus características, rasgos peculiares $\mathrm{y} / \mathrm{o}$ diferenciadores relacionados con la muestra a estudiar en un solo momento de tiempo.

La fiabilidad del instrumento de medición se mide a través del estadístico Alpha de Cronbach que es un valor entre 0 y 1 , el cual está dado por la siguiente formula:

$$
\alpha=\frac{k}{k-1}\left[1-\frac{\sum S_{i}^{2}}{S_{t}^{2}}\right]
$$

Para validar el instrumento se tomó una muestra piloto de 100 graduados, donde se aplicó la encuesta, la cual costa de 54 preguntas, dividida en 4 componentes. El proceso de validación se realizó en el software estadístico SPSS versión 24, usándose las variables policotómicas; lográndose los resultados que se muestran en la tabla 1.

Tabla 1. Estadísticas de fiabilidad

\begin{tabular}{ccc}
\hline Alfa de Cronbach & Alfa de Cronbach basada en elementos estandarizados & N de elementos \\
\hline 0,789 & 0,916 & 56 \\
\hline Fuente: Elaboración propia & &
\end{tabular}

Del resultado del coeficiente Alpha de Cronbach que se muestra en la Tabla 1 , se obtuvo un valor de 0.789 ; teniendo en cuenta que el criterio de evaluación es que este valor sea superior a 0.7 , se concluye que el instrumento de medición es adecuado en cuanto a su consistencia interna y por tanto arrojará resultados confiables.

Población y muestra. La población para esta investigación está determinada por los graduados de los últimos 5 años de grado, es decir, las cohortes 2013-1 hasta 20172. A partir de ella, se realizó un muestreo probabilístico estratificado con un nivel de confianza del $95 \%$ y un nivel de error del $5 \%$, donde se divide la población objeto de estudio en subgrupos, dando como resultado, el listado de los programas académicos de la modalidad presencial de la CECAR.

De acuerdo al tamaño muestral de los grupos, se calculó el porcentaje de cada programa académico perteneciente a este estudio, proporcional al tamaño de la población de los programas listados, y posterior se saca el tamaño de la muestra de cada uno de los programas, para un total de una muestra general de 548 graduados.

Otra población con la cual se trabaja es con empleadores y/o sector externo, para ello se toman de referencia las empresas con las cuales se tiene convenio de prácticas y 
que tengan graduados laborando, esta es una muestra intencional a partir de la base de datos que se tienen en la Universidad, desde la oficina de Relaciones Interinstitucionales y la oficina de Seguimiento a graduados.

Consideraciones éticas. De acuerdo con el estudio realizado, se consideró como "investigación con riesgo mínimo" de acuerdo al Decreto 11, Resolución No 008430. Santafé de Bogotá (1993). La participación en el estudio fue totalmente voluntaria, previa autorización a través de la aceptación y firma de un consentimiento informado por parte de los participantes. Los resultados se utilizaron solo para fines investigativos preservando los principios de integridad e intimidad de las personas. Toda la información obtenida y los resultados de la investigación fueron tratados confidencialmente y archivados en papel y medio electrónico.

\section{RESULTADOS}

Análisis descriptivo de las variables sociodemográficas por facultad.

Tabla 2. Estadísticos demográficos por facultad.

\begin{tabular}{|c|c|c|c|c|c|c|}
\hline Variable & Categoría & $\begin{array}{c}\text { Ciencias Básicas, } \\
\text { Ingenierías y } \\
\text { Arquitectura }\end{array}$ & $\begin{array}{c}\text { Ciencias } \\
\text { Económicas y } \\
\text { administrativas }\end{array}$ & $\begin{array}{l}\text { Derecho } \\
\text { y Ciencias } \\
\text { Políticas }\end{array}$ & $\begin{array}{l}\text { Humanidades } \\
\text { y Educación }\end{array}$ & Total \\
\hline \multirow{2}{*}{ Edad } & Promedio & 29,0 & 35,9 & 35,4 & 33,4 & 34,0 \\
\hline & Desviación Estándar & 4,3 & 9,1 & 9,4 & 9,3 & 9,0 \\
\hline \multirow{2}{*}{ Género } & Hombre & $65,0 \%$ & $50,6 \%$ & $45,6 \%$ & $38,6 \%$ & $46,4 \%$ \\
\hline & Mujer & $35,0 \%$ & $49,4 \%$ & $54,4 \%$ & $61,4 \%$ & $53,6 \%$ \\
\hline \multirow{2}{*}{ Estado Civil } & Casado(a)/Unión Libre & $43,3 \%$ & $57,1 \%$ & $50,0 \%$ & $61,8 \%$ & $56,4 \%$ \\
\hline & Soltero & $56,7 \%$ & $42,9 \%$ & $50,0 \%$ & $38,2 \%$ & $43,6 \%$ \\
\hline \multirow{3}{*}{$\begin{array}{l}\text { Nro. De } \\
\text { Hijos }\end{array}$} & No tiene & $63,3 \%$ & $38,2 \%$ & $42,2 \%$ & $44,3 \%$ & $44,2 \%$ \\
\hline & Entre 1 y 2 & $33,3 \%$ & $48,8 \%$ & $50,0 \%$ & $45,2 \%$ & $45,8 \%$ \\
\hline & 3 o más & $3,3 \%$ & $12,9 \%$ & $7,8 \%$ & $10,5 \%$ & $10,0 \%$ \\
\hline \multirow{4}{*}{ Escolaridad } & Profesional & $58,3 \%$ & $64,7 \%$ & $40,0 \%$ & $62,3 \%$ & $58,9 \%$ \\
\hline & Especialización & $26,7 \%$ & $25,3 \%$ & $47,8 \%$ & $21,9 \%$ & $27,7 \%$ \\
\hline & Maestría & $15,0 \%$ & $10,0 \%$ & $10,0 \%$ & $14,0 \%$ & $12,2 \%$ \\
\hline & Doctorado & $0,0 \%$ & $0,0 \%$ & $2,2 \%$ & $1,8 \%$ & $1,1 \%$ \\
\hline \multirow{6}{*}{ Vivienda } & En arriendo & $31,7 \%$ & $24,7 \%$ & $30,0 \%$ & $35,1 \%$ & $30,7 \%$ \\
\hline & $\begin{array}{l}\text { De un familiar sin } \\
\text { pagar arriendo }\end{array}$ & $40,0 \%$ & $26,5 \%$ & $26,7 \%$ & $25,0 \%$ & $27,4 \%$ \\
\hline & $\begin{array}{l}\text { De un tercero sin } \\
\text { pagar arriendo }\end{array}$ & $0,0 \%$ & $1,2 \%$ & $0,0 \%$ & $0,9 \%$ & $0,7 \%$ \\
\hline & $\begin{array}{l}\text { Propia y la está } \\
\text { pagando }\end{array}$ & $6,7 \%$ & $23,5 \%$ & $16,7 \%$ & $15,4 \%$ & $17,2 \%$ \\
\hline & $\begin{array}{l}\text { Propia totalmente } \\
\text { pagada }\end{array}$ & $20,0 \%$ & $22,4 \%$ & $17,8 \%$ & $19,3 \%$ & $20,1 \%$ \\
\hline & Otra & $1,7 \%$ & $1,8 \%$ & $8,9 \%$ & $4,4 \%$ & $4,0 \%$ \\
\hline
\end{tabular}

Fuente: Elaboración propia.

En la Tabla 1 se condensan las estadísticas descriptivas, discriminadas por facultad. Determinado que el $41.6 \%$ de los egresados provienen de la facultad de Humanidades y Educación, además la población de estudio presentó una edad promedio de 34 años, siendo la facultad de ciencias básicas la que arrojó la edad promedio más baja; en relación al género, estuvo compuesta de forma general por un $53.6 \%$ de mujeres y el 
46.4\% de hombres, así mismo se encontró que la facultad de humanidades y educación es mayoritariamente de mujeres $(61.4 \%)$, en contraposición a ciencias básicas con predominio de hombres $(65 \%)$.

En relación al estado civil el $56.4 \%$ tiene algún tipo de vínculo, mientras el restante $43.6 \%$ indica que son solteros. El $44.2 \%$ de los egresados no tiene hijos, un $45.8 \%$ tiene entre uno y dos. De forma global se establece que solo el $41.1 \%$ de los egresados ha realizado algún postgrado, siendo la facultad de Derecho y ciencias políticas la que se destaca en ese sentido (60\%). En general el $27.7 \%$ de los postgrados corresponden a especializaciones, un $12.2 \%$ a maestrías, mientras un $1.1 \%$ a doctorados.

\section{Dimensión. Percepción de los graduados con respecto a las competencias académicas y laborales de los graduados.}

Tabla 3. Distribución del nivel de satisfacción de las competencias generales.

\begin{tabular}{|c|c|c|c|c|}
\hline Competencia & Muy insatisfecho & Insatisfecho & Satisfecho & Muy satisfecho \\
\hline Exponer las ideas por medios escritos & $67(12,2 \%)$ & $13(2,4 \%)$ & $283(51,6 \%)$ & $185(33,8 \%)$ \\
\hline Comunicarse oralmente con claridad & $73(13,3 \%)$ & $19(3,5 \%)$ & $244(44,5 \%)$ & $212(38,7 \%)$ \\
\hline Persuadir y convencer a sus interlocutores & $71(13 \%)$ & $29(5,3 \%)$ & $276(50,4 \%)$ & $172(31,4 \%)$ \\
\hline $\begin{array}{l}\text { Identificar y utilizar símbolos para comunicarse (lenguaje icónico, lenguaje } \\
\text { no verbal, etc.) }\end{array}$ & $63(11,5 \%)$ & $66(12 \%)$ & $283(51,6 \%)$ & $136(24,8 \%)$ \\
\hline Aceptar las diferencias y trabajar en contexto multiculturales & $71(13 \%)$ & $16(2,9 \%)$ & $259(47,3 \%)$ & $202(36,9 \%)$ \\
\hline $\begin{array}{l}\text { Utilizar herramientas informáticas básicas (procesadores de texto, } \\
\text { hojas de cálculo, correo electrónico, etc.) }\end{array}$ & $69(12,6 \%)$ & $43(7,8 \%)$ & $265(48,4 \%)$ & $171(31,2 \%)$ \\
\hline Aprender y mantenerse actualizado & $72(13,1 \%)$ & $31(5,7 \%)$ & $247(45,1 \%)$ & $198(36,1 \%)$ \\
\hline Ser creativo e innovador & $72(13,1 \%)$ & $27(4,9 \%)$ & $246(44,9 \%)$ & $203(37 \%)$ \\
\hline Buscar, analizar, administrar y compartir información & $73(13,3 \%)$ & $21(3,8 \%)$ & $261(47,6 \%)$ & $193(35,2 \%)$ \\
\hline Crear, investigar y adoptar tecnología & $62(11,3 \%)$ & $57(10,4 \%)$ & $272(49,6 \%)$ & $157(28,6 \%)$ \\
\hline Diseñar e implementar soluciones con el apoyo de tecnología & $58(10,6 \%)$ & $65(11,9 \%)$ & $274(50 \%)$ & $151(27,6 \%)$ \\
\hline Identificar, plantear y resolver problemas & $66(12 \%)$ & $20(3,6 \%)$ & $269(49,1 \%)$ & $193(35,2 \%)$ \\
\hline Capacidad de abstracción análisis y síntesis & $71(13 \%)$ & $24(4,4 \%)$ & $280(51,1 \%)$ & $173(31,6 \%)$ \\
\hline Comprender la realidad que lo rodea & $69(12,6 \%)$ & $18(3,3 \%)$ & $242(44,2 \%)$ & $219(40 \%)$ \\
\hline Asumir una cultura de convivencia & $71(13 \%)$ & $15(2,7 \%)$ & $233(42,5 \%)$ & $229(41,8 \%)$ \\
\hline Asumir responsabilidades y tomar decisiones & $70(12,8 \%)$ & $13(2,4 \%)$ & $221(40,3 \%)$ & $244(44,5 \%)$ \\
\hline
\end{tabular}

Fuente: Elaboración propia.

Al indagar acerca de la satisfacción de los graduados con respecto a las competencias genéricas adquiridas se encontró que todas tuvieron altos porcentajes de calificación en los niveles Satisfechos y Muy satisfechos (Conjuntamente porcentajes mayores a 73\%). Los mayores porcentajes de satisfacción se obtuvieron en los ítems "Exponer las ideas por medios escritos, Identificar y utilizar símbolos para comunicarse y Capacidad de abstracción análisis y síntesis" con un 51.6\% simultáneamente. En cuanto a nivel satisfacción con respecto a estas competencias en el nivel con una calificación de muy insatisfecho en mayor proporción el $13.3 \%$ se siente insatisfecho con las competencias Comunicarse oralmente con claridad, Buscar, analizar, administrar y compartir información, Identificar, plantear y resolver problemas. Este insumo es sumamente valioso para adaptar los currículos de acuerdo a las necesidades y percepciones de los graduados. 
Díaz-Theran et al - Horizonte académico de los graduados, modalidad presencial CECAR

Tabla 4. Distribución del nivel de satisfacción de las competencias laborales.

\begin{tabular}{lcccc}
\hline \multicolumn{1}{c}{ Competencia } & Muy insatisfecho & Insatisfecho & Satisfecho & Muy satisfecho \\
\hline $\begin{array}{l}\text { Planificar y utilizar el tiempo de manera efectiva de tal forma } \\
\text { que se logran los objetivos planeados }\end{array}$ & $72(13,1 \%)$ & $22(4 \%)$ & $273(49,8 \%)$ & $181(33 \%)$ \\
$\begin{array}{l}\text { Utilizar herramientas informáticas especializadas } \\
\text { (paquetes estadísticos, software de diseño, etc.) }\end{array}$ & $67(12,2 \%)$ & $67(12,2 \%)$ & $277(50,5 \%)$ & $137(25 \%)$ \\
Formular y ejecutar proyectos & $65(11,9 \%)$ & $78(14,2 \%)$ & $262(47,8 \%)$ & $143(26,1 \%)$ \\
Trabajar en equipo para alcanzar metas comunes & $72(13,1 \%)$ & $23(4,2 \%)$ & $246(44,9 \%)$ & $207(37,8 \%)$ \\
Trabajar de manera independiente sin supervisión permanente & $67(12,2 \%)$ & $20(3,6 \%)$ & $265(48,4 \%)$ & $196(35,8 \%)$ \\
Aplicar valores y ética profesional en el desempeño laboral & $69(12,6 \%)$ & $12(2,2 \%)$ & $221(40,3 \%)$ & $246(44,9 \%)$ \\
Adaptarse a los cambios (trabajar en contextos nuevos y diversos) & $69(12,6 \%)$ & $16(2,9 \%)$ & $235(42,9 \%)$ & $228(41,6 \%)$ \\
Trabajar bajo presión & $66(12 \%)$ & $55(10 \%)$ & $251(45,8 \%)$ & $176(32,1 \%)$ \\
Promedio (Total) & $6.6 \%$ & $12 \%$ & $46 \%$ & $34 \%$ \\
\hline
\end{tabular}

Fuente: Elaboración propia.

En la Tabla 4 se condensan la percepción que tienen los encuestados con relación a las competencias más fuerte y más débil que poseen, así como también la más y menos útil en desarrollo de su labor. Se observa que con mayor porcentaje el $46 \%$ de los evaluados se siente satisfecho con las competencias laborales con una mayor proporción en la competencia "Utilizar herramientas informáticas especializadas (paquetes estadísticos, software de diseño, etc.)" y en menor porcentaje en promedio el $12 \%$ de los evaluados se encuentra muy insatisfecho con las competencias laborales adquiridas durante su formación y el $6.6 \%$ insatisfecho.

\section{Dimensión: Percepción de los graduados con respecto a las fortalezas y debilidades adquiridas.}

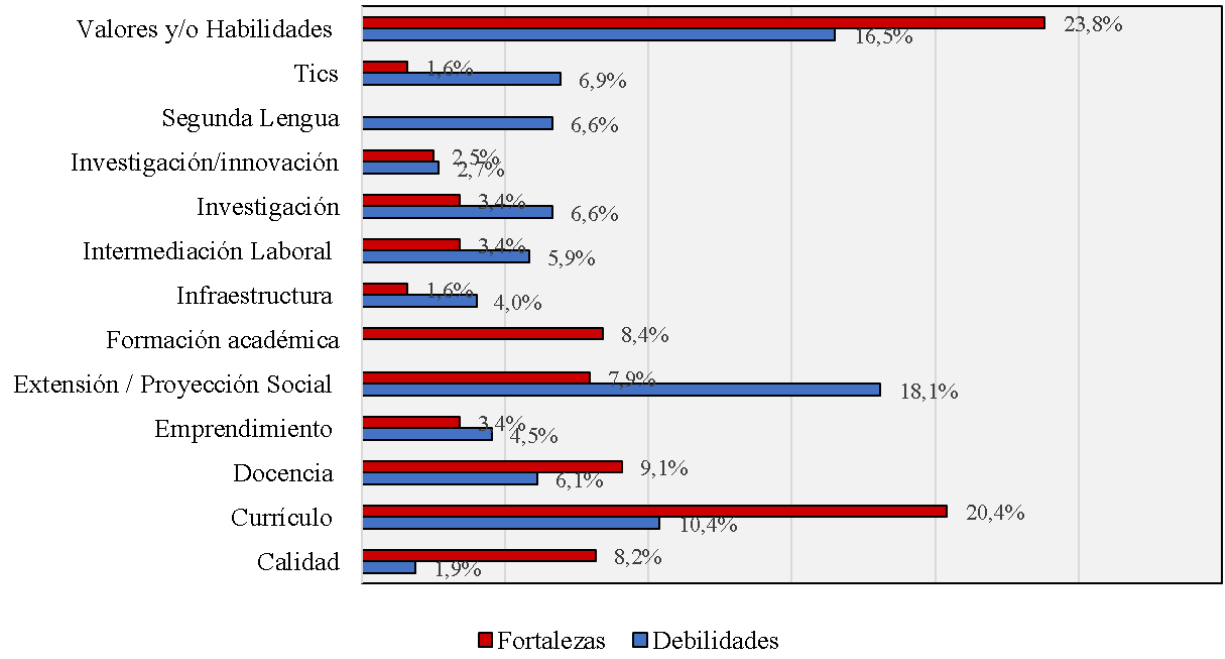

Gráfica 1. Distribución de la percepción de fortalezas y debilidades Fuente: Elaboración propia

Con respecto a las principales fortalezas que presentan los graduados de CECAR, Ios egresados manifestaron que fueron los valores o habilidades $(23.8 \%)$, el currículo $(20.4 \%)$ y la formación académica (8.4\%). De la misma forma, en relación a las debilidades afirmaron que principalmente fue la falta de extensión o proyección social $(18.1 \%)$, pero también los valores o habilidades $(16.5 \%)$ y el currículo $(10.4 \%)$. En la gráfica 1 se detallan todas las fortalezas y debilidades. 


\section{Dimensión: Percepción de los graduados con respecto a la calidad de la institución}

Los $51.8 \%$ de egresados manifestaron que volverían debido a la calidad de la formación, un $19.3 \%$ por la calidad de los docentes, mientras que un $17.7 \%$ por los recursos de apoyo que brinda la institución en el proceso de formación (Tabla 5).

Tabla 5. Distribución de las razones para volver a estudiar en la institución.

\begin{tabular}{ccc}
\hline Razón & Frecuencia & Porcentaje \\
\hline Calidad de la formación & 263 & $51,8 \%$ \\
Calidad de los profesores & 98 & $19,3 \%$ \\
Fundamentación para crear empresa & 22 & $4,3 \%$ \\
Los recursos de apoyo al proceso de formación & 90 & $17,7 \%$ \\
Reconocimiento de la institución & 35 & $6,9 \%$ \\
\hline
\end{tabular}

Fuente: Elaboración propia.

Dentro de los egresados que manifestaron el interés de volver, se encontró el 48.6\% mostraron interés por la realización de una especialización, el 38.7\% una maestría, el $6.6 \%$ un diplomado, un 3.3\% un seminario o curso, solo el $2.7 \%$ expresó el deseo de hacer una nueva carrera (Tabla 6).

Tabla 6. Distribución del tipo de estudio a realizar.

\begin{tabular}{ccc}
\hline Tipo de Estudio & Frecuencia & Porcentaje \\
\hline Pregrado & 14 & $2,7 \%$ \\
Seminario/curso & 17 & $3,3 \%$ \\
Especialización & 250 & $48,6 \%$ \\
Diplomado & 34 & $6,6 \%$ \\
Maestría & 199 & $38,7 \%$ \\
\hline
\end{tabular}

Fuente: Elaboración propia.

Se clasificaron 56 temáticas de interés, sin embargo, las cinco que mostraron mayor demanda fueron Educación y Tics (8.3\%), Docencia Universitaria (5.1\%), Administración Pública (4.9\%), Gerencia del talento humano (4.2\%), Gestión ambiental (3.8\%) y Entrenamiento deportivo (3.8\%).

En la Gráfica 2 se exhiben los principales temas de interés por parte de los egresados.

En la Gráfica 3 se observa que $71.2 \%$ de los egresados mostraron el deseo de volver a estudiar en la institución con alto sentido de pertinencia, mientras que un $2.0 \%$ afirmó que no. De la misma forma un $20.4 \%$ mostró un nivel de pertenencia media.

\section{Dimensión: Reconocimiento académico y laboral de los graduados}

Del conjunto de personas que fueron destacadas, se puedo establecer que el $37 \%$ fue a causa de su desempeño laboral, el $23.1 \%$ debido a su labor docente, el $9.8 \%$ por motivos de su gestión social o ambiental, un $8.7 \%$ gracias a un buen rendimiento académico o en sus trabajos de grado o en los resultados de las pruebas Saber Pro, mientras un $6.9 \%$ por ser innovadores o emprendedores (Tabla 7). 
Díaz-Theran et al - Horizonte académico de los graduados, modalidad presencial CECAR

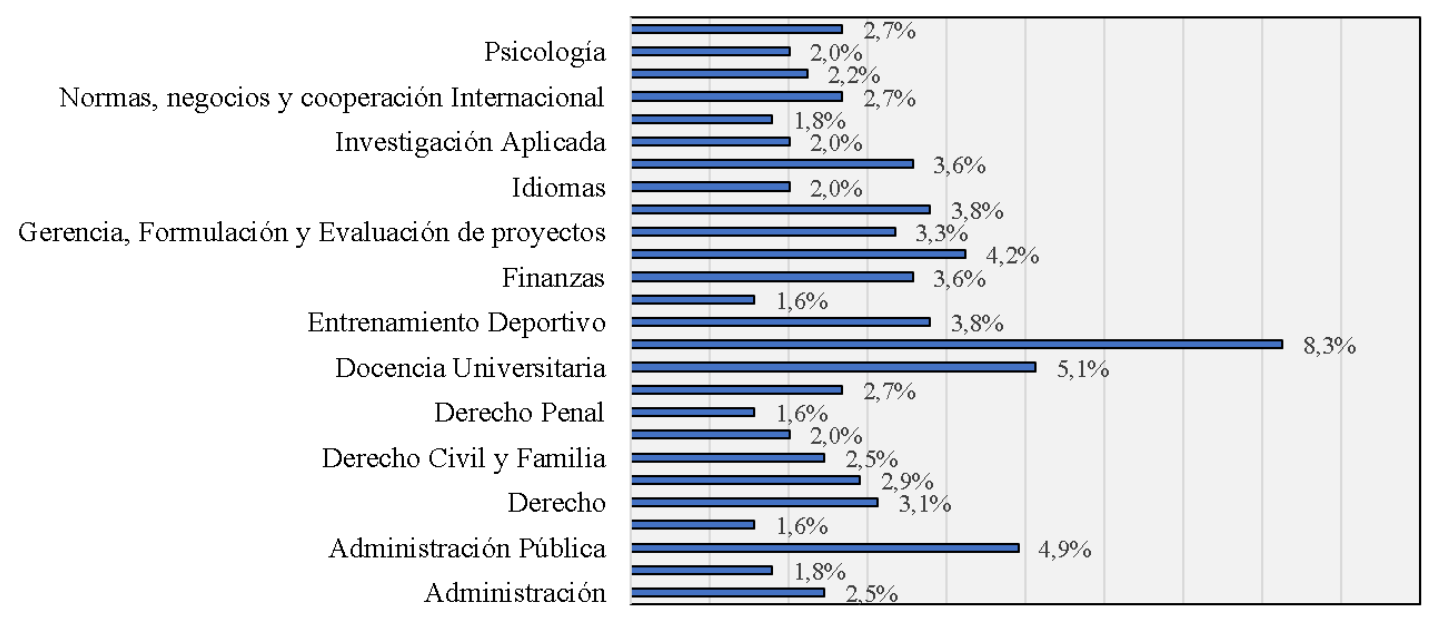

Gráfica 2. Principales temas de Interés Fuente: Elaboración propia.

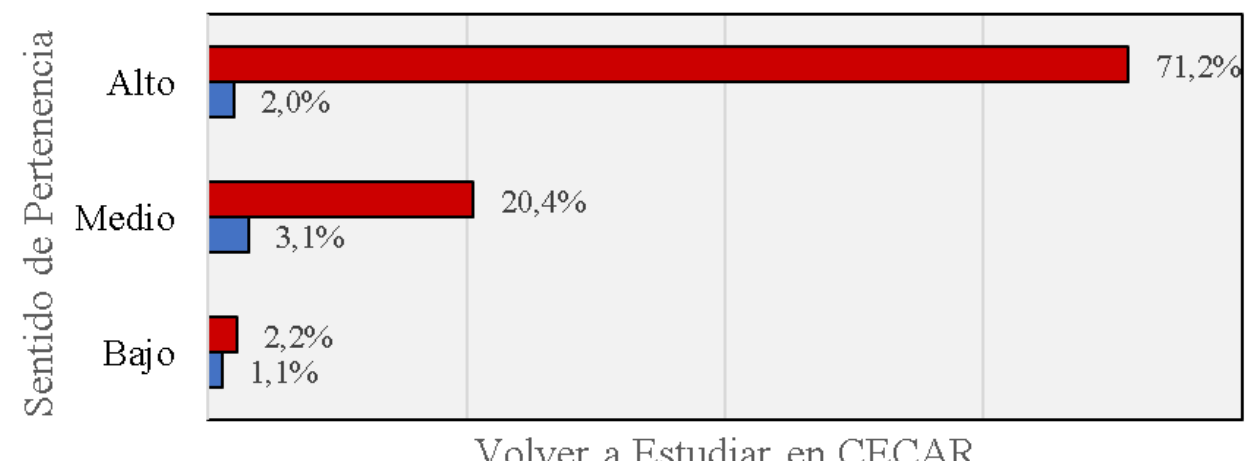

$\square \mathrm{Si}$ 口No

Gráfica 3. Distribución de la pertenencia respecto a regresar a estudiar. Fuente: Elaboración propia.

Tabla 7. Distribución de las razones de reconocimiento.

\begin{tabular}{ccc}
\hline Motivo de Reconocimiento & Frecuencia & Porcentaje \\
\hline Aportes en Cultura / Deporte & 11 & $6,4 \%$ \\
Desempeño Laboral & 64 & $37,0 \%$ \\
Emprendimiento / Innovación & 12 & $6,9 \%$ \\
Gestión en Proyectos & 4 & $2,3 \%$ \\
Gestión Social / Ambiental & 17 & $9,8 \%$ \\
Investigación / Ponencia / Publicación & 10 & $5,8 \%$ \\
Labor Docente & 40 & $23,1 \%$ \\
Rendimiento Académico / Tesis / Ecaes & 15 & $8,7 \%$ \\
\hline Total & 173 & $100,0 \%$ \\
\hline
\end{tabular}

Fuente: Elaboración propia 
En la gráfica 4 se exhiben las principales entidades que concedieron reconocimientos a los egresados. El 32.9\% de los graduados no específico, el $23.7 \%$ fueron exaltados por instituciones de educación superior, el $12.1 \%$ por establecimientos estatales de orden nacional, un $11.6 \%$ por entes gubernamentales locales, mientras que un $6.4 \%$ de instituciones educativas. En relación a la vinculación científica se determinó que solo un $13.1 \%$ de los egresados pertenece a algún tipo de asociación

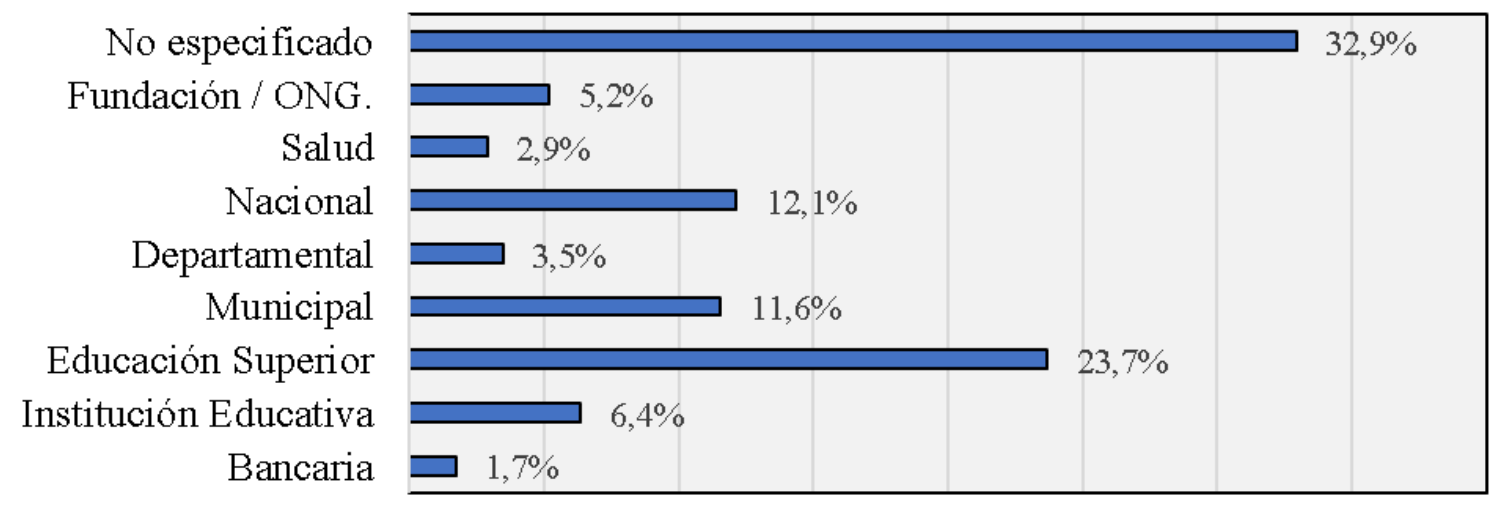

Gráfica 4. Distribución de entidades que otorgan reconocimiento. Fuente: Elaboración propia.

\section{Dimensión: Percepción del impacto académico de los graduados en el medio.}

Finalmente, al indagar sobre la percepción de los egresados con respecto a la calidad de formación brindada por la institución, se encontró que el 55.5\% considera que alta, mientras un $30.7 \%$ muy alta. Así mismo el $53.6 \%$ de ellos considero que hay una alta concordancia entre los conocimientos adquiridos durante su formación y las competencias del mercado de trabajo, mientras un $30.1 \%$ percibe que es muy alta.

El $48.8 \%$ de los graduados de CECAR piensa que la institución le aporta un alto desarrollo social a la región y un $38.9 \%$ considera que es muy alto. Un $48.5 \%$ expresaron que los graduados satisfacen las necesidades de trabajo de forma alta y un $25.5 \%$ de manera muy alta. Así mismo el $50.7 \%$ considera que los programas ofertados responden altamente a las necesidades sociales de la región y un $27.6 \%$ de forma muy alta (Grafica 5).

La diversidad de la oferta educativa de CECAR permite responder a las necesidades sociales de la región

Las necesidades del mercado de trabajo se satisfacen con los graduados de CECAR

CECAR como Institución de Educación Superior le aporta al desarrollo social de la región

Concordancia entre los conocimientos adquiridos durante su formación y las competencias del mercado de trabajo

Calidad en la formación brindada por CECAR

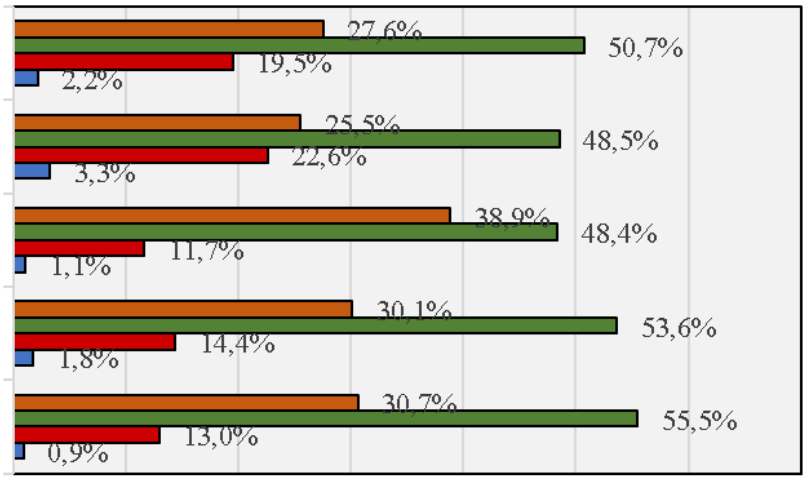

$\square$ Muy alta $\square$ Alta $\square$ Media $\square$ Baja

Gráfica 5. Distribución de percepciones de aspectos de interés.

Fuente: Elaboración propia. 
Díaz-Theran et al - Horizonte académico de los graduados, modalidad presencial CECAR

\section{DISCUSIÓN}

La mirada externa e interna de los procesos académicos de los programas de pregrado son parámetros que denotan la calidad de la percepción de los servicios profesionales, por lo que realizar un análisis contextualizado de la realidad es de gran importancia, para esto se consultaron distintas investigaciones que anteceden a esta y le brindan un corpus teórico practico que permita una interpretación amplia de los resultados expuestos.

Los resultados de este proceso científico, explica en detalle cual es el horizonte académico de los graduados de la Corporación Universitaria del Caribe CECAR, utilizando un muestreo estratificado por facultades para darle participación a los programas académicos del pregrado de la Corporación, otra de las características de este abordaje, es destacar que en mayor proporción los graduados perciben un nivel de satisfacción alto con respecto a las competencias generales y laborales adquiridas durante su formación con porcentajes en promedio superiores al 50\%. Esta situación es similar a la observada en el estudio realizado por Maya Guerra y Herrera Herrera (2011) en la Fundación Universitaria Católica del Norte, quienes encontraron una percepción de satisfacción de los egresados y de los empleadores, el cual permitió evidenciar la pertinencia, la calidad de sus programas y la alineación de sus servicios educativos frente al propósito misional, esta recurrió a una indagación secundaria para conocer información de contexto sobre situaciones que pudieran ser comparables; también, a una búsqueda primaria mediante encuestas electrónicas sobre una muestra estadística estratificada de la población.

La indagación en la Fundación Universitaria Católica del Norte permitió obtener resultados de variación en aspectos de tipo demográfico, laboral y académico de los graduados, así como la percepción de satisfacción con el programa respectivo y los servicios de la Institución por parte de graduados y empleadores. Los resultados de factor académico arrojaron que un $70 \%$ de los empleadores tiene una percepción superior o sobresaliente sobre el desempeño laboral del graduado de la Universidad Católica del Norte.

En cuanto a la dimensión percepción de los graduados con respecto a la calidad de la institución, en este estudio se encontró que el $51.8 \%$ de egresados manifestaron que volverían debido a la calidad de la formación recibida en la institución, seguido de la calidad en la formación de los docentes, además el 93.8\% de los egresados mostraron el deseo de volver a estudiar en la corporación demostrando un alto sentido de pertenencia con ella. Dato similar se encuentra en el análisis realizado por los investigadores Chamorro, Paz Peña y Vernaza Pinzon (2009), donde la percepción con respecto a la calidad y la satisfacción recibida por el egresado obtuvo una calificación considerada buena en su mayoría, destacando la trayectoria y el prestigio del programa y la institución y confirmando las expectativas de ingreso de los estudiantes. Esto denota la importancia que tiene los procesos misionales dentro de la identificación del perfil profesional y laboral de estudiantes desde un contexto específico y que contribuya a las necedades del entorno, iniciando un trabajo acorde a las necesidades desde los primeros semestres.

Asimismo, la investigación con la que se compara los resultados obtenidos en la actualidad son los aportados por los autores Ruiz, Jaraba y Romero Santiago (2008) en su estudio denominado la formación en psicología y las nuevas exigencias del mundo laboral realizada a 60 graduados para determinar las competencias laborales exigidas al psicólogo en el contexto laboral, esta investigación se realizó en la ciudad de Barranquilla donde los resultados más relevante destacan la importancia que tienen las competencias específicas de la profesión para la formación de un profesional, además del manejo de herramientas tecnológicas, habilidades obligatorias que facilitan el trabajo en las condiciones actuales, así como solucionar problemas, tener iniciativa y ciertas actitudes que muestren la calidad humana y ética del profesional; este dato es similar al hallado 
en el presente estudio donde los encuestados destacan en mayor proporción con una evaluación de satisfechos y muy satisfechos. Los mayores porcentajes de satisfacción se obtuvieron en exponer las ideas por medios escritos, aplicar valores y ética profesional en el desempeño laboral y asumir responsabilidades y tomar decisiones.

En cuanto a la dimensión reconocimiento, este documento presenta resultados favorables que no discrepan en lo académico donde se evidencio por medio de las encuestas aplicadas que el $37 \%$ de los graduados que se encuentran en condición de empleabilidad recibió un reconocimiento a causa de su desempeño laboral, el $23.1 \%$ debido a su labor docente, el $9.8 \%$ por motivos de su gestión social o ambiental, un $8.7 \%$ gracias a un buen rendimiento académico o en sus trabajos de grado o en los resultados de las pruebas Saber Pro, mientras un $6.9 \%$ por ser innovadores o emprendedores. Dentro de los tipos de reconocimientos recibidos por los egresados, se destaca principalmente una exaltación en público a través de un certificado o diploma (54.3\%), una mención de honor (13.3\%), y certificado con constancia en su hoja de vida (9.2\%). Este dato es de importancia institucional porque reporta el impacto que los graduados tienen en el medio y brinda una perspectiva de la calidad de la formación que se ofrece.

Asimismo, Gaspar Domingos, Capó Pérez y Lombillo Rivero (2015), manifiestan la importancia de utilizar metodologías para el seguimiento de los graduados, que redunden en los procesos de autoevaluación y autorregulación de los programas, encontrándose resultados similares a los hallados actualmente, dado que a lo que se refiere a las competencias adquiridas el $87 \%$ de los docentes y $94 \%$ de los empleados escogieron como satisfactorio la relación positiva entre las competencias generales adquiridas y las requeridas por el empleo.

Otro antecedente es el denominado estudio de impacto de graduados para el fortalecimiento de los programas académicos Por medio del estudio de Impacto de graduados, la Corporación Universidad de la Costa (CUC) a través de la Facultad de Ingeniería da a conocer una perspectiva de sus graduados y empleadores. Se utiliza la metodología de tipo descriptivo para la recopilación y actualización de la información requerida, a través de una encuesta estructurada con base en el Observatorio Laboral para la Educación. Se evidencia en los resultados que los graduados son conscientes de la necesidad de formación tanto en un segundo idioma como en estudios complementarios a los programas de pregrado, y poseen un alto nivel de pertinencia con la institución, también se encontró que el estudio realizado permite tener una visión más amplia del desempeño de los graduados de la universidad, identificando principales competencias y necesidades requeridas por el mercado laboral (Pulido Roncancio, Mercado Caruso, y Obredor Baldovino, 2014).

De esta manera, el reto de los programas de pregrado es establecer las condiciones académicas acordes a las necesidades del contexto, generar de esta forma un impacto en la sociedad, cumplir con el eje misional de acuerdo con los lineamientos de calidad en educación superior e incentivar desde los estudios de impacto métodos de cambio y de restructuración acordes a las demandas y necesidades que contribuyan la realización de procesos de autoevaluación y autorregulación.

\section{Agradecimientos}

A los distintos programas académicos de pregrado de la Corporación Universitaria del Caribe CECAR. 


\section{REFERENCIAS}

Apunte sobre el término graduado Ministerio de Educación Nacional. Recuperado de: http://www.mineducacion.gov.co/sistemasdeinformacion/1735/w3-article-254707. $\underline{\text { html }}$

Beneitone, P., Esquetini, C., González, J., Maletá, M., Siufi, G., Wagennar, R. (ed). (2004 - 2007). Reflexiones y perspectivas de la educación superior en américa latina. Recuperado de http://tuning.unideusto.org/tuningal/index.php?option=com docmanyItemid $=191$ ytask =view categoryycatid $=22$ yorder $=$ dmdate publishedyascdesc $=$ DESC

Briones, G. (1996). Epistemología de las ciencias sociales. Obtenido de https://www. insumisos.com/lecturasinsumisas/Epistemologia $\% 20$ de $\% 201$ as $\% 20$ ciencias $\% 20$ sociales.pdf

Buunaaisie, C., Manyara, A., Annett, H., Pájaro, E., Bray, Y., Ige, J., ... Evans, D. (2018). Empleabilidad y experiencias profesionales de graduados internacionales de Maestría en Salud Pública: un estudio de métodos mixtos. Public Health, 62-69. doi: https:// doi.org/10.1016/j.puhe.2018.03.032

Corporación Universitaria del Caribe - CECAR, Acuerdo de Junta № 17 de agosto de 2014.

Gaspar Domingos, A., Capó Pérez, J. R., y Lombillo Rivero, I. (2015). Variables e indicadores para seguimiento de graduados del instituto superior politécnico de Kwansa Sul, Angola. Ciencias Técnicas Agropecuarias, 52-56.

Hernández Sampieri, R., y Mendoza Torres , C. P. (2018). Metodología de la Investigación. Las rutas cualitativa, cuantitativa y mixta. Mexico: Mc Graw Hill Educactión.

Jaramillo Quintero, N. Y., Montesino Acosta, K. L., Suárez Tamara, D. C., Vergara Arroyo, V. M., y Martínez Royert, J. C. (Septiembre de 2018). Opinión de los empleadores sobre las competencias de los egresados del programa de enfermería de la Universidad de Sucre frente a los retos cotidianos que enfrentan en el desempeño de sus funciones. Sincelejo: Universidad de Sucre.

Maya Guerra, J. I., y Herrera Herrera, M. E. (2011). Caracterización e impacto de los graduados de la Catolica del Norte Fundación Universitaria. Universidad Católica del Norte, 78-112.

Ministerio de Educación Nacional (2007) Seguimiento a egresados, información para la Pertinencia. Recuperado de http://www.mineducacion.gov.co/cvn/1665/w3article-136997.html

Paternina Avilez, Y. E., y Reyes Oviedo, K. L. (2011). Competencias laborales requeridas a los psicólogos egresados de la Corporación Universitaria del Caribe CECAR. Sincelejo: Repositorio CECAR.

Paternina Avilez, Y. E., Chagüi Hernández, A. J., Castro Vargas, M. A., \& Buitrago Domínguez, M. (2015). La formación en psicología: una mirada desde los estudiantes. Búsqueda, 2(14), 84-95. https://doi.org/10.21892/01239813.61 
Pérez-Contreras, B., González-Otero, K., \& Polo-Bolaño, Y. (2018). Perfil sociodemográfico y económico de estudiantes universitarios. Búsqueda, 5(20), 48-62. https://doi. org/10.21892/01239813.391

Pulido Roncancio, C. G., Mercado Caruso, N. N., y Obredor Baldovino, T. P. (2014). Estudio de Impacto de Graduados para el fortalecimiento de los programas academicos. INGE CUC, 19-29.

Ruiz, M., Jaraba, B., y Romero Santiago, L. (2008). La formación en psicología y las nuevas exigencias del mundo laboral: Competencias laborales exigidas a los psicólogos. Psicología desde el Caribe, 136-157.

Sanabria Navarro, J., Silveira Pérez, Y., y Diaz Theran, K. M. (2017). Toma de Decisiones Científicas en las Universidades. Sincelejo: CECAR.

Sistema Nacional de Acreditación (2013). Lineamientos para la acreditación de programas de Pregrado. Recuperado de http://www.cna.gov.co/1741/articles-186359 pregrado 2013.pdf

Tirado, R., Tejeda, R., y Cedeño, G. (2015). Implementación institucional de un modelo cooperativo para el seguimiento a graduados en Ecuador. Revista de la educación superior, 44(173), 125-156. Recuperado de https://ac-els-cdn-com. ezproxy.cecar.edu.co:2443/S0185276015000667/1-s2.0-S0185276015000667main.pdf? tid=d8afbcb4-fcf6-47fc-b8f5-282a9931e372yacdnat $=1530808531$ e699f3a7cf9d73b9c74e4eac4d480db2

Universidad Nacional de Colombia (10 de Junio de 2011). El egresado de la educación superior, el fruto de la gestión academica e investigativa de las universidades . Obtenido de https://agenciadenoticias.unal.edu.co/uploads/media/Claves Digital No. 47.pdf

Wilches, E.C., Muñoz, V., Carvajal, N., y Segura, A. (2016). Characterization and perceived impact on graduates of a specialization in cardiopulmonary physiotherapy at a public university in the south west of Colombia. Periodo 2009-2013. Revista Ciencias de la Salud, 14(1), 43-52. Recuperado de https://www-scopus-com. ezproxy.cecar.edu.co:2443/record/display.uri?eid=2-s2.0-84958074117yorigin=re sultslistysort $=$ plf-fysrc $=$ syst $1=$ university + graduates + in + colombiayst $2=y$ sid $=$ cab8 $\underline{5665 e 7 b 97 d b 4 d 2 d 475 b a 2207 d c a 1 y s o t=b y s d t=b y s l=47 y s=T I T L E-A B S-K E Y \% 28 u n i}$ versity+graduates+in+colombia $\% 29 y$ relpos $=15$ yciteCnt $=0$ ysearchTerm $=$ 